\title{
Effect of Core Size on Nonlinear Transmission in Silicon Optical Fibers
}

\author{
P. Mehta ${ }^{1}$, N. Healy ${ }^{1}$, T.D. Day ${ }^{2}$, P.J.A. Sazio ${ }^{1}$, J.V. Badding $^{2}$, and A.C. Peacock ${ }^{1}$ \\ 1. Optoelectronics Research Centre, University of Southampton, Southampton SO17 1BJ, United Kingdom \\ 2. Department of Chemistry and Materials Research Institute, Pennsylvania State University 16802 PA, USA \\ acp@orc.soton.ac.uk
}

\begin{abstract}
The nonlinear transmission properties of two hydrogenated amorphous silicon fibers with core diameters of $5.7 \mu \mathrm{m}$ and $1.7 \mu \mathrm{m}$ are characterized. The measured Kerr nonlinearity, two-photon absorption and free-carrier parameters will be discussed in relation to device performance.
\end{abstract}

(C) 2011 Optical Society of America

OCIS codes: $060.2270,060.2290,060.4370$.

\section{Introduction}

Silicon waveguides are an ideal platform for nonlinear optical signal processing due to their high nonlinearity, as well as the high core/silica-cladding index contrast which allows for tailoring of the waveguide dispersion. The incorporation of silicon into the optical fiber geometry provides an important step towards integrating this technology with more conventional fiber infrastructures. However, the silicon fibers fabricated to date have typically had large core sizes [1], so that high peak powers are required to observe nonlinear transmission [2]. Despite this, we have recently demonstrated all-optical modulation using two-photon absorption (TPA) in a hydrogenated amorphous silicon (a-Si:H) fiber with a $\sim 6 \mu \mathrm{m}$ core, though the device speed was limited by the long free-carrier lifetime associated with the micron sized core [3]. The scaling of the fiber core sizes down towards those approaching the dimensions typically employed in on-chip waveguides is thus critical for the development of low power, high speed devices as well as opening up the possibility for dispersion tailoring.

In this paper we demonstrate nonlinear transmission in the smallest core $(1.7 \mu \mathrm{m})$ silicon optical fiber to date and compare the nonlinear parameters to those of a larger $5.7 \mu \mathrm{m}$ diameter core. Due to the reduced effective mode area, we show an enhancement of nonlinear absorption and self-phase modulation (SPM) in the smaller core structure for much lower input peak powers. Importantly this is also accompanied by a significant reduction in the free-carrier lifetime which paves the way for ultrafast optical processing. The multimode behavior of the fibers is investigated via Fourier analysis of the imaged output, revealing that for both fibers most of the light is contained in the fundamental mode.

\section{Theory and Experiment}

The silicon core fibers are fabricated using a high pressure chemical fluid deposition technique [2]. The deposition parameters such as precursor concentration, pressure, and furnace temperature differ between fibers of different core diameters thereby potentially giving rise to a variation in the deposited material quality and the corresponding parameters. The linear losses determined via cut-back measurements are $0.9 \mathrm{~dB} / \mathrm{cm}$ and $3 \mathrm{~dB} / \mathrm{cm}$ for the large and small core fibers, respectively, which are indicative of the relative material qualities.
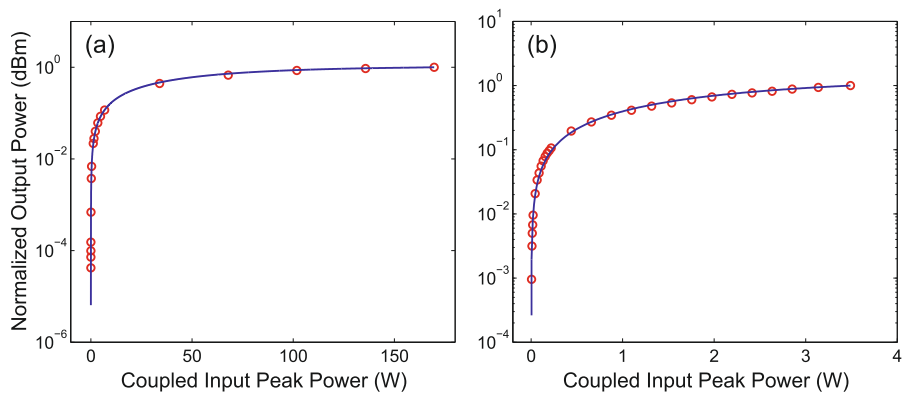

Fig. 1. Nonlinear absorption in a-Si:H core fibers with diameters of (a) $5.7 \mu \mathrm{m}$. and (b) $1.7 \mu \mathrm{m}$. 
The nonlinear transmission properties of the fibers are characterized by first measuring the nonlinear losses due to TPA $\beta_{\mathrm{TPA}}$, free-carrier absorption (FCA) $\sigma_{\mathrm{FCA}}$, as well as the corresponding FC lifetime $\tau$, and then by observing pulse broadening through self-phase modulation (SPM) to estimate the Kerr nonlinearity parameter $n_{2}$. For both set-ups a high power mode-locked $1540 \mathrm{~nm}$ laser was used with $700 \mathrm{fs}$ (fwhm) pulses at $40 \mathrm{MHz}$. Fig. 1 shows the nonlinear loss measurements for the (a) $5.7 \mu \mathrm{m}$ and (b) $1.7 \mu \mathrm{m}$ core fibers. The fitted curves are obtained using a simplified propagation equation for the temporal evolution of the pulse governed by the effects of loss [2], from which we obtain, (a) $\beta_{\mathrm{TPA}} \sim 0.8 \mathrm{~cm} / \mathrm{GW}, \sigma_{\mathrm{FCA}} \sim 1 \times 10^{-16} \mathrm{~cm}^{2}$ and (b) $\beta_{\mathrm{TPA}} \sim 0.83 \mathrm{~cm} / \mathrm{GW}, \sigma_{\mathrm{FCA}} \sim 1 \times 10^{-16} \mathrm{~cm}^{2}$. We attribute the slightly higher value of the TPA parameter to the difference in the material quality evident from the higher linear loss of the small core fiber. The carrier lifetime of the two fibers can then be determined using simple pump-probe measurements [3], and we have found that this is significantly reduced in the small core fiber $\tau \sim 6 \mathrm{~ns}$, vs $\tau \sim 200 \mathrm{~ns}$ in the larger core, allowing for faster device speeds.

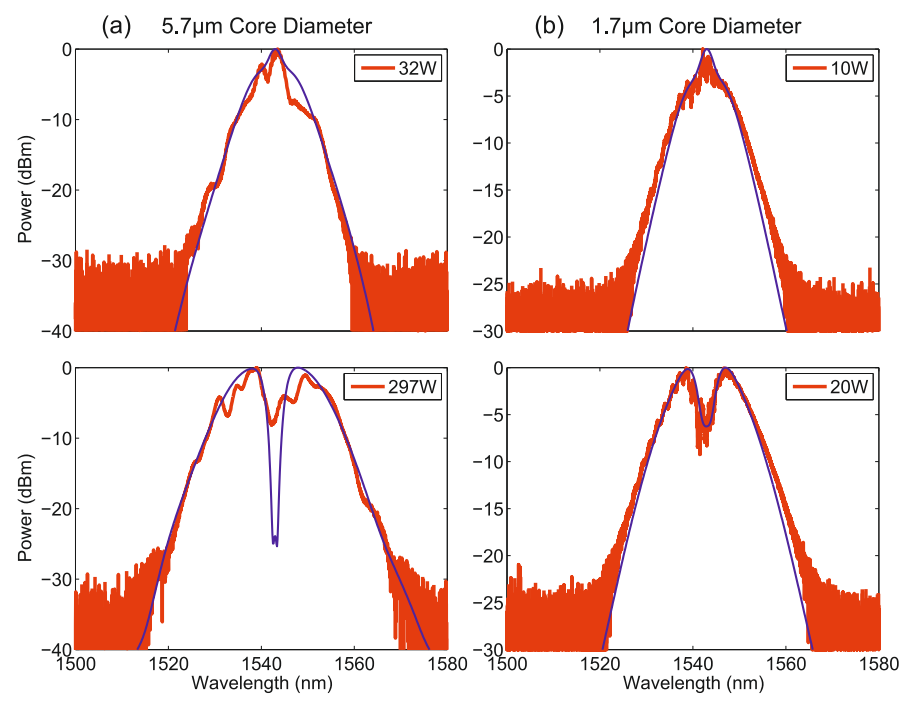

Fig. 2. Nonlinear transmission in silicon core fibers.

The spectral evolution of the pulses in the two fibers is then shown in Fig. 2, where (a) $5.7 \mu \mathrm{m}$ and (b) $1.7 \mu \mathrm{m}$. These results clearly highlight the different input peak powers required to obtain similar levels of broadening. Nonlinear transmission in the silicon core fiber is modeled using a modified form of the nonlinear Schrödinger equation (NLSE) [2]. Using the predetermined loss parameters, the output pulse spectra can be fitted to estimate $n_{2}$. From the blue outlined fits to the red experimental spectra in Fig. 2, we obtain (a) $n_{2} \sim 1.5 \times 10^{-13} \mathrm{~cm}^{2} / \mathrm{W}$ and (b) $n_{2} \sim 1.8 \times$ $10^{-13} \mathrm{~cm}^{2} / \mathrm{W}$. Again, we attribute the higher value of the nonlinearity in the small core fiber to the difference in the material quality. The oscillations that appear on the spectra are due to the multimode nature of the waveguides. However, Fourier analysis of the intermodal interference indicates that the higher order mode contribution is very small, particularly in the smaller core fiber, with only a small percentage of the propagating power contained in these modes. This will be discussed in relation to the dispersion properties of the two fibers.

\section{Conclusion}

By scaling the core diameter of silicon optical fibers to smaller dimensions, we have demonstrated an enhancement in the nonlinearity and a reduction of the free-carrier lifetime, providing a route to faster, lower power nonlinear fiber devices.

\section{References}

1. J. Ballato, T. Hawkins, P. Foy, B. Yazgan-Kokuoz, C. McMillen, L. Burka, S. Morris, R. Stolen, R. Rice, "Advancements in semiconductor core optical fiber," Opt. Fiber Technol. 16, 399-408 (2010).

2. P. Mehta, N. Healy, N. F. Baril, P. J. A. Sazio, J. V. Badding, and A. C. Peacock, "Nonlinear transmission properties of hydrogenated amorphous silicon core optical fibers," Opt. Express 16, 16826-16831 (2010).

3. P. Mehta, N. Healy, T. D. Day, J. R. Sparks, P. J. A. Sazio, J. V. Badding, and A. C. Peacock, "All-optical modulation using two-photon absorption in silicon core optical fibers," Opt. Express 19, 19078-19083 (2011). 